The Astrophysical Journal SupPlement Series, 90:683-688, 1994 February

(c) 1994. The American Astronomical Society. All rights reserved. Printed in U.S.A.

\title{
MICROWAVE BURST TIMESCALES AND SOLAR FLARE ACCELERATION PROCESSES
}

\author{
A. KRÜGER, B. KLIEM, AND J. HildebrandT \\ Astrophysical Institute Potsdam, D-14482 Potsdam, Germany \\ AND \\ V. V. ZAITSEV \\ Institute of Applied Physics, Russian Academy of Sciences, Nizhnij Novgorod, 603600, Russia \\ Received 1993 February 23; accepted 1993 August 18
}

\begin{abstract}
The occurrence of quite different timescales in solar microwave bursts is considered and possible ways of their physical interpretation are discussed. An interesting feature is the existence of hierarchic time structures, an example of which is provided by the solar event of 1991 March 23.

Plasma parameter sets to be invoked for the interpretation of timescales are estimated on the base of different models such as the collisionless conduction front model, the twisted magnetic loop model, the coalescence model, and the electric circuit model. With emphasis on the interpretation of burst fine structures with timescales of a few seconds the coalescence model has been favored. On the other hand, the simultaneous occurrence of a large range of different timescales appears well suited to be described by the circuit model.
\end{abstract}

Subject headings: acceleration of particles — Sun: flares — Sun: radio radiation

\section{INTRODUCTION}

It is widely accepted that electrons accelerated or heated by flares in the solar corona are most directly detected through their gyromagnetic emission and collisional bremsstrahlung in the microwave range as well as by collisional bremsstrahlung in the hard X-ray region. Both emissions supply complementary information about the underlying process(es) of energization of the radiating particles. In this paper we will focus on the microwave emission and discuss possible hints on the flare process provided by the timescales of the radiation in light of recent observations and theoretical approaches, which are briefly reviewed. The blurring of short time structures in the production of fast electrons, due to propagation of the electrons between the acceleration site and the radiation source, is presumably less severe for the microwaves which are produced at least partly in the corona than for the hard X-rays emanating from the chromospheric layers. In this paper the term microwave emission refers to the direct, single-particle radiation in the centimeter- and millimeter-wave range and should be distinguished from collective emission (type III-like emission and usually highly polarized spikes) which typically occurs in the decimetric region but sometimes extends into the centimetric range. The temporal structure of the collective emissions reflects both the production rate of energetic electrons and the characteristic scales of the underlying kinetic instability.

\section{CHARACTERISTIC TIMESCALES OF SOLAR MICROWAVE BURST RADIATION}

Early studies of solar radio bursts already established a wide distribution of burst durations (Covington 1958) which may range from fractions of seconds to hours. Separated by morphological criteria, two large categories of microwave bursts, viz., impulsive and gradual bursts, have been roughly distinguished. But the validity of the early simple explanation, in which impulsive burst phenomena are due to acceleration processes and gradual bursts are caused by plasma heating, is still questionable. Later on bursts have been discussed consisting of several timescales. Sturrock et al. (1984) described four characteristic timescales of subseconds, few seconds, few minutes, and tens of minutes present in microwave bursts. However, as the authors are aware, comprehensive systematic statistical studies on different burst timescales are rather rare. Evidence for a (quasi-) quantization of solar millimeter-bursts by a study of repetition rates of superposed spiky burst elements have been found by Kaufmann et al. (1980). Reviews of different phases of burst development and time structures can be found, for example, in Krüger ( 1979) and in Kundu \& Vlahos (1982).

Concluding from catalogs of millimeter-wave observations (see, e.g., Urpo, Pohjolainen, \& Teräsranta 1992), the temporal structures of the bursts show a broad range of scales. Different types of structures are visible which can vary from event to event and sometimes combine to form very complex flux histories. The rising part of a burst frequently consists of numerous steps with much steeper flux increase than the envelope, subpulses may recur at a certain repetition rate, flux pulsations can occur, second and subsecond spikes are found to be superposed on an otherwise slowly varying emission. We emphasize that for individual bursts the scales of the temporal structures do not appear to form a continuum, rather they group into different ranges of scales. As a typical example we consider the event of 1991 March 23, shown in Figure 1, selected here due to the availability of digital multifrequency observations with high time resolution.

One can distinguish a sequence or "hierarchy" of different timescales in several ranges:

$(1) \approx 10$ minute duration of the main burst phase,

(2) 1-2 minute duration of the two main burst pulses, 


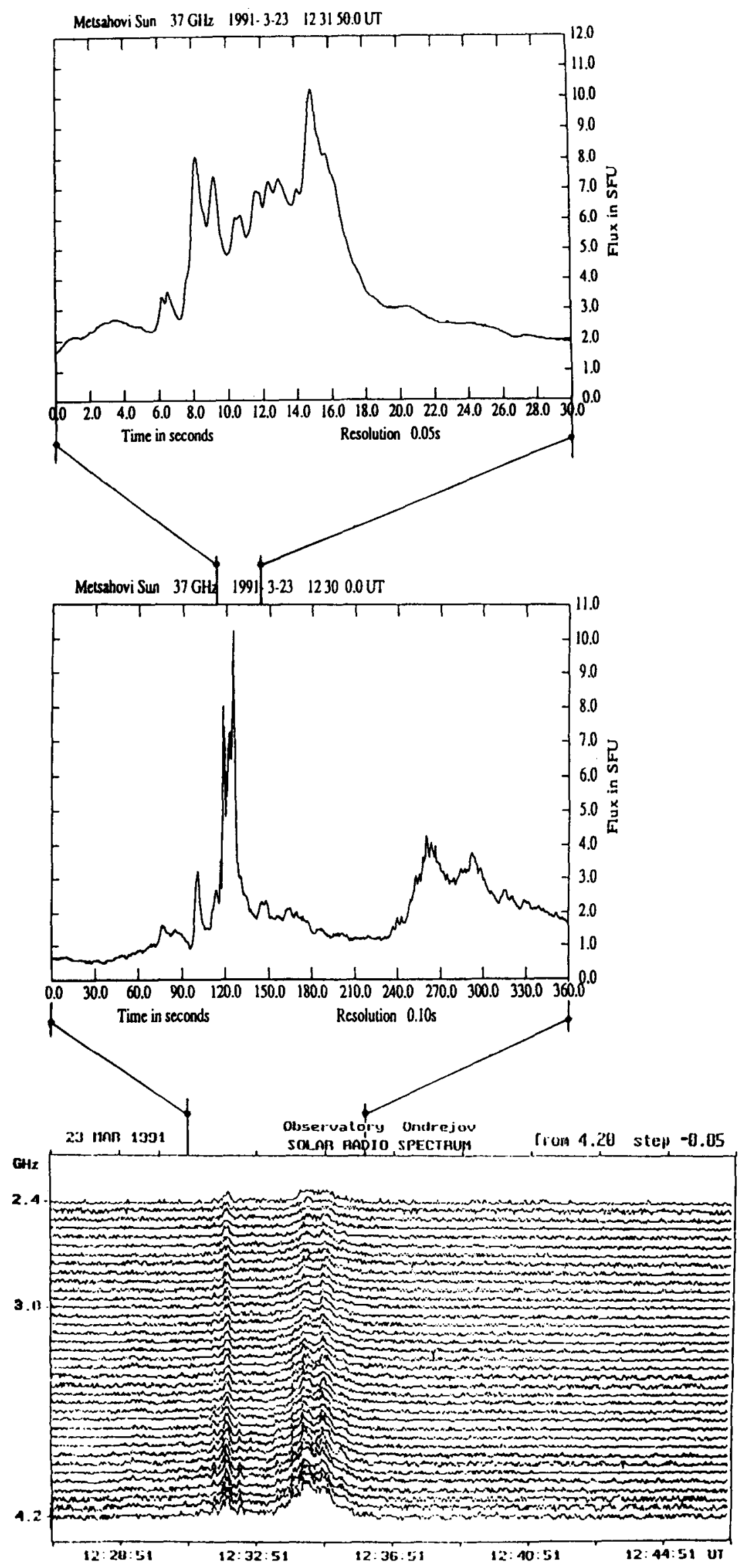

FIG. 1.-Display of different timescales by the microwave burst on 1991 March 23. Bottom: Spectrographic record of the Ondrejov Observatory (from Tlamicha et al. 1991); middle and top: $37 \mathrm{GHz}$ records of the Metsähovi Radio Research Station (from Urpo et al. 1992). 
$(3) \approx 3$ s duration of subpulses during the second part of the event,

(4) $\lesssim 1 \mathrm{~s}$ duration of spiky burst elements occurring in the first part of the burst event.

Moreover, $a \gtrsim 20$ minute relaxation time of the whole event is evident in the more sensitive records of the Tremsdorf Observatory not shown in Figure 1. A statistics of timescales will be the topic of a subsequent paper.

Among the different timescales the search for very short scales is of special interest. Such timescales are indicated by the occurrence of radio spikes and pulsations. But the existence of structures with timescales much less than $1 \mathrm{~s}$ at millimeter waves seems to be an open question. Although the occurrence of such structures has been reported in the literature ( e.g., Hurford et al. 1979; Kaufmann et al. 1980, 1986; Takakura et al. 1983; Krüger et al. 1987) one must keep in mind that their verification requires, due to the influence of interferences, simultaneous observations at separated sites.

The remarkable fact that the burst time structures are not randomly distributed is also indicated by a wavelet analysis of the burst time profile, which is currently being pursued. Preliminary results show a difference of burst structures from a Gaussian distribution and a scaling behavior. A more detailed description of these time series analyses will be published separately.

The occurrence of temporal fine structures at a timescale clearly separated from the scale of the main burst pulse corresponds to the hypothesis that the flare energy release consists of a possibly large number of elementary steps, that is, proceeds in a fragmentary manner. In the following we attempt to relate the different timescales to various theoretical models for energy release and particle energization in solar flares. Our emphasis is on short timescales and the primary, impulsive energy release which sets the strongest requirements on the mechanism of particle acceleration.

\section{COMPETING FLARE MODELS EXPLAINING MICROWAVE BURST TIMESCALES}

We will compare four models for the impulsive flare phase which encompass most of the basic magnetic structures and energy release mechanisms discussed so far and should therefore be representative for the majority of proposed models. In these models, the timescale of the energetic particle production and subsequent growth of radio emission is related to different physical processes, and consequently the timescales which may be explained are different.

\subsection{Collisionless Conduction Fronts}

We start with the thermal flare model (Brown, Melrose, \& Spicer 1979), quantitatively analyzed by Batchelor et al. (1985), in which the hot plasma, produced by the primary energy release in a coronal loop, is confined by collisionless conduction fronts propagating at the ion acoustic speed, $c_{s}$, toward the loop foot points. The basic structure is a magnetic loop, and the primary energy release mechanism is not necessarily specified. Microwave and hard X-ray emissions are produced within the confined hot plasma and, to a minor extent, at the loop foot points by electrons escaping through the con- duction fronts. The rise time of the microwave burst, $\tau_{r}$, does therefore not directly reflect the timescale of the primary energy release but is determined by the growth of the volume of hot plasma,

$$
\tau_{r} \approx l /\left(2 c_{s}\right)
$$

where $l$ is the length of the loop. For average burst sources $\left(T_{e}=10^{7}-10^{8} \mathrm{~K}, l=10^{9}-10^{9.5} \mathrm{~cm}\right)$, we find $\tau_{r} \approx 5-55 \mathrm{~s}$. This is comparable with typical rise times of microwave bursts (Fig. 1). Also, shorter timescales may be obtained in this model by considering smaller loop lengths $l$ (Batchelor 1987, 1989, 1990); this corresponds then simply to smaller (less energetic) flares. But the model is not well suited to explain substantial fluctuations of the flux at the second scale during the impulsive rise of complex flares, as seen in the beginning of the first main pulse in Figure 1 (top panel at about $8 \mathrm{~s}$ from origin).

\subsection{Twisted Magnetic Loop Model}

In nonthermal models of the impulsive flare phase, the rise of the microwave intensity directly reflects the production of mildly relativistic electrons, which in turn is confined mainly to the action (duration) of the primary flare instability. Sturrock et al. (1984) proposed the unwinding of a twisted "elementary flux tube" (EFT) as the elementary energy release process (or flare instability). The basic structure is again a magnetic loop. They assumed that the unwinding occurs basically at the Alfvenic timescale and thus found for the unwinding time

$$
\tau_{u} \approx l / v_{\mathrm{A}} .
$$

For the coronal part of the flux tube, with field strengths $B \approx$ 100-200 G, density $N_{e}=10^{9.5} \mathrm{~cm}^{-3}$, and values of the loop length $l$ as above, unwinding times $\tau_{u} \approx 1-10 \mathrm{~s}$ result. This is in the range of durations of subpulses and spiky elements in impulsive microwave bursts.

For energetical reasons, one EFT cannot account for a whole burst, and a bundle of EFTs must be involved in the energy release of the impulsive phase in this model, which corresponds to the fragmentation hypothesis. Sturrock et al. (1984) suppose that the unwinding process propagates across a bundle of EFTs with velocity $R_{E} \tau_{u}^{-1}$ where $R_{E}$ is the radius of an EFT. The timescale of the impulsive energy release (and the microwave burst rise time) is then given by

$$
\tau_{I}=\left(R_{B} / R_{E}\right) \tau_{u},
$$

where $R_{B}$ is the radius of the bundle of EFTs. With $R_{E}=$ $10^{9} B_{[\text {Gauss }}^{-1 / 2} \mathrm{~cm}$ (Sturrock et al. 1984) and $R_{B} \approx l / 2$ one obtains $\tau_{I}=10-160 \mathrm{~s}$.

One should note, however, that it has not yet been shown how the unwinding of a whole EFT can proceed at the (fast) Alfvénic timescale. The unwinding implies anomalous dissipation of the flux tube current and diffusion of the field, or field line reconnection. The anomalous dissipation is highly hypothetical because it is unclear how the current can be concentrated into narrow channels to reach supercritical density, it involves much smaller spatial scales, and the timescales of a 
kinetic current-driven instability. The interaction of the smallscale processes with the magnetohydrodynamical evolution has not yet been explored, so we will not include this possibility in our discussion. The reconnection of magnetic field lines proceeds in general at a hybrid time scale between the Alfvénic and resistive timescales, $\tau_{R} \sim S^{1 / 2} \tau_{\mathrm{A}}$ (based on the appropriate length scale which should be of order $R_{E}$ ), where $S$ is the magnetic Reynolds number $\left(S \sim 10^{12}\right.$ for our chosen parameter values $)$. This yields timescales a factor of $\left(R_{E} / l\right) S^{1 / 2} \sim 3 \times$ $10^{4}$ larger than above. Fast reconnection at the Alfvénic timescale has been found only for special conditions which do not correspond to the geometry of EFTs (see the following section ). We therefore regard the unwinding of EFTs as probably too slow in comparison with the timescales found in impulsive microwave bursts. Our arguments are supported by recent results of numeric simulations by Dahlburg, Antiochos, \& Zang (1991) which showed that unwinding of elementary flux tubes is too slow to apply to flares.

\subsection{Coalescence Model}

Shorter timescales have been found in a nonthermal model where the impulsive energy release again involves a reconnection process but the geometry is that of a current sheet containing current filaments (resembling a pair of, or even multiple flux tubes). If these tubes possess the same sense of field line twist, the attracting force between the tubes drives the coalescence instability which is basically an instability of ideal MHD and thus operates at the Alfvénic timescale in its initial phase of flux tube approach. Complete coalescence of the flux tubes requires reconnection of field lines and proceeds in general also at a hybrid timescale $\sim S^{1 / 2} \tau_{\mathrm{A}}$ (Pritchett \& Wu 1979), where now the current sheet half-width enters as characteristic spatial scale. However, the reconnection is driven here by the attracting current filaments and may be faster. Tajima, Brunel, \& Sakai (1982), Leboeuf, Tajima, \& Dawson (1982), and Bhattacharjee, Brunel, \& Tajima ( 1983) indeed found fast coalescence; in their two-dimensional simulations complete merging of pairs or of multiple flux tubes occurred in less than two Alfvén times if the plasma was compressible (i.e., plasma $\beta \ll 1$ and guide field component $B_{z} \ll B_{y}$ where $B_{y}$ denotes the antiparallel field component outside the sheet ) and the current density profile of the flux tubes was sufficiently peaked. Here the Alfvén time is based on $l_{y}$, the largest distance between the coalescing filaments. The propagation velocity of the coalescence process in $y$-direction reaches the Alfvén velocity under these circumstances (in marked difference to the case of unwinding of flux tubes). We will suppose the existence of fast coalescence in flares because the condition of compressibility can be easily met in the corona (where $\beta \ll 1$ ): in current sheets formed by newly emerging flux the old and the new flux systems must be inclined by a large angle $(>\pi / 2)$ - a case frequently observed. Since fast coalescence has subsequently been observed in several numerical experiments, the condition on the current density profile does not appear to be a strongly restrictive requirement. In three dimensions one may expect that the process is initiated by a disturbance at a certain point, where it is locally characterized by the timescale $\sim l_{y} v_{\mathrm{A}}^{-1}$, and then extends in $z$-direction typically with Alfvén velocity. Since $l_{z} \gg l_{y}$ in order to release energy through coalescence, we obtain the characteristic timescale of the duration of impulsive energy release in this model as

$$
\tau_{\mathrm{CI}} \sim l_{z} v_{\mathrm{A}}^{-1}
$$

One can expect $l_{z}$ to be somewhat smaller than, but of the order of, typical loop lengths. With $l_{z}=10^{8.5}-10^{9} \mathrm{~cm}$ and values of $B$ and $N_{e}$ as above, we find $\tau_{\mathrm{Cl}} \sim 0.4-3 \mathrm{~s}$.

This corresponds to the shortest timescales in our sample event and also to typical small-scale structures in HXRs. These HXR structures with scales of the order of few to $\gtrsim 10 \mathrm{~s}$ were termed "elementary flare bursts" (de Jager \& de Jonge 1978), but recent observations with higher sensitivity and resolution (Aschwanden, Benz, \& Schwartz 1993) indicate that elementary HXR pulses may have durations of the order of $\$ 0.5 \mathrm{~s}$.

We note that also the coalescence model is consistent with the fragmentation hypothesis: multiple filaments in a current sheet are naturally produced by a tearing instability, and the coalescence then proceeds via a sequence of elementary coalescence events of neighboring filament pairs (e.g., Tajima et al. 1987; Kliem 1988). With an extension of $l_{z} \sim 10^{7} \mathrm{~cm}$ for the elementary energy release region, that is, coalescing flux-tube length (as suggested for example by decimetric spike burst bandwidths; Benz 1985), the coalescence timescale becomes $\sim 20 \mathrm{~ms}$ in agreement with the shortest temporal structures observed so far in microwave bursts (see Kaufmann et al. 1980, 1986). The model has been considered for a broader range of parameters by Krüger, Kliem, \& Hildebrandt (1988).

Particles are accelerated in the electric fields induced by the coalescing flux tubes with the characteristic timescale $\tau_{\mathrm{CI}}$ (Kliem 1994) or by shock waves which propagate out of the region of coalescence (Sakai \& Ohsawa 1987).

\subsection{Circuit Model}

An alternative process of flare energy release is related to the interaction of field-aligned currents with filament matter in the frame of the circuit current disruption model of Alfvén \& Carlquist (1967) (see also Zaitsev \& Stepanov 1991, 1992). In comparison with the processes of tearing and coalescence, a smaller portion of the released energy goes to particle and fluid acceleration here, and heating is the dominant energization process. Subsequently, the acceleration proceeds in the tail of the energy distribution by a runaway process (Holman 1985; Holman \& Benka 1992). Alternatively, double layers may form in coronal circuits and accelerate the particles.

\subsubsection{Scenario and Rise Time of Energy Release}

The general scenario of energy release in a circuit solar flare model is as follows (Zaitsev \& Stepanov 1991, 1992): in a magnetic loop an electric current $I=10^{11}-10^{12} \mathrm{~A}$ is presumed to be generated by photospheric convection. The lifetime of this current is of the order of the inductivity rise time $\tau_{L} \approx$ $L(d L / d t)^{-1}$ of the equivalent electric circuit along the magnetic loop and photosphere, that is, of the order of the time of magnetic flux emergence. This time usually ranges from several hours to several days. A flare can be triggered by a prominence which lies above the magnetic loop (or loop system). If the mass of the prominence becomes sufficiently large, the flute instability of the ballooning mode arises (Pustilnik 
1973). The cold, partly ionized prominence plasma penetrates into the current channel of the magnetic loop at a characteristic time

$$
\tau_{\mathrm{fl}} \approx r / v_{T_{i}} \approx 1-10 \mathrm{~s}
$$

where $r$ is the loop radius and $v_{T_{i}}$ the thermal velocity of ions. The penetration of the cold plasma into the current channel switches on an effective dissipation mechanism which is connected with ion-atom collisions in a nonstationary plasma (Cowling 1957). As a consequence the nonlinear resistance of the channel $R(I)$ grows up to a magnitude $R(I) \approx 3 \times 10^{-2} \Omega$ corresponding to a heating rate of $W=R I^{2} \approx 10^{27}-10^{28}$ ergs $\mathrm{s}^{-1}$.

Since electric field components parallel to the magnetic field are present in the magnetic loop, a part of the electrons is accelerated by the runaway effect. Thus in the frame of this model the energy of accelerated electrons is only a part of the whole flare energy.

\subsubsection{Duration of the Flare Process and Energy Fragmentation}

The duration of the flare process is determined by the characteristic timescale of the electric current dissipation in the equivalent circuit after the current disruption

$$
\tau_{0} \approx L / R \approx 500-5000 \mathrm{~s},
$$

where we use the inductance $L \approx 10 \mathrm{H}$ calculated by Alfvén $\&$ Carlquist (1967) for a slender semicircular flux tube with a length $\sim 10^{9} \mathrm{~cm}$ and thickness $\sim 2 \times 10^{8} \mathrm{~cm}$. This time is of the order of the duration of the flaring process taking place in a magnetic loop or loop system.

However, the energy release can be fragmented, for example, in the form of "subflares." The latter is connected with the heating of the cold, dense plasma of the prominence which enters the current channel. Due to heating as a result of nonlinear resistance the plasma becomes totally ionized and the energy release is interrupted. The duration of a "subflare" is of the order

$$
\tau_{\mathrm{sf}} \approx N_{e} \kappa T / q \approx 10-100 \mathrm{~s}
$$

for a particle density $N_{e} \approx 10^{12} \mathrm{~cm}^{-3}$, temperature $T \approx 10^{5}-$ $10^{6} \mathrm{~K}$, and an energy dissipation rate $q \approx 1-10 \mathrm{ergs}^{-3} \mathrm{~cm}^{-1}$. Such "subflaring" can be repeated by the penetration of new, cold, and dense prominence matter into the current channel, for example, if the prominence fulfils quasi-periodic oscillations. In that case "subflaring" occurs during phases of the compression of the magnetic loop. The oscillation period, and accordingly the repetition time of the "subflares,"

$$
\tau_{\text {osc }}=\frac{2 \pi}{3^{1 / 4}}\left(\frac{g}{l}+\frac{2 \kappa T}{m_{i} l d}\right)^{-1 / 2}
$$

can vary from $10^{2}$ to $10^{3} \mathrm{~s}$ depending on the parameters of the system ( $l$ is the length of the magnetic loop, $d$ is the prominence diameter, $g$ is the gravitational acceleration, $\kappa$ is Boltzmann's constant, and $m_{i}$ is the ion mass).

\subsubsection{Fine Structures of Radiation}

In addition to a long range of timescales from $10 \mathrm{~s}$ to $1 \mathrm{hr}$ in this model, a hierarchy of timescales can be noted which may be related to the fine structure of the flare process. Alfvénic and fast magneto-acoustic oscillations of the magnetic flux tube excited during the impulsive flare phase can modulate the flow of energetic electrons in the flux tube due to the modulation of the ratio $B_{\max } / B_{\min }$ and also modulate the electron streams penetrating into the chromosphere (Roberts, Edwin, \& Benz 1984; Zaitsev \& Stepanov 1989). The characteristic timescales of such modulations are $\tau_{\mathrm{A}} \approx l / v_{\mathrm{A}} \approx 10 \mathrm{~s}$ and $\tau_{\mathrm{FMS}} \approx d / v_{\mathrm{A}} \approx$ $1 \mathrm{~s}$, respectively. Oscillating plasma instabilities and processes of quasi-linear relaxation can also lead to a modulation of electron streams and of the energy density of plasma wave with a timescale $\tau \approx \Lambda / \nu_{e i}-\lambda / \gamma \approx 10^{-1}$ to $10^{-4} \mathrm{~s}$, where $\Lambda$ is the logarithm of the ratio between the energy density of plasma waves in the source to the plasma wave energy density in the equilibrium state. These short timescales can occur in fine structures of microwave, X-ray, and gamma-ray bursts. It must be mentioned that short timescales also exist in other flare scenarios ( see $\S 3.3$ ). The circuit flare model, however, explains a series of timescales which are often observed.

\section{DISCUSSION AND CONCLUSIONS}

In this paper attention is paid to the occurrence of different timescales in solar microwave bursts and their relevance to the underlying flare-particle energization process. The dominant timescales shown in $\S 2$ are related to

1. The burst duration (lifetime),

2. The duration and the repetition rate of major peaks of a complex-burst emission,

3. The rise times or durations of steplike flux increases of which the envelope of an impulsive flux increase is often composed,

4. Time characteristics of spiky or fluctuating burst emission occurring, for example, during the maximum phase of a burst peak.

These features describe a sequence or "hierarchy" of timescales which do not form a continuum but group into different ranges of scales, at least in one and the same event. This suggests that the bursts consist of "bricks" corresponding to elementary energization processes. Our discussion of the hierarchy of timescales, and in particular the shortest time scales, in light of various theoretical approaches to the impulsive flare energy release (and particle acceleration) problem favors the coalescence model and the current circuit model. The collisionless conduction front model has difficulties to explain regular structuring of subpulses and fine structures of emission at the $\$ 1 \mathrm{~s}$ scale, and the twisted magnetic loop model probably acts too slowly.

In the frame of the coalescence model the burst profile is due to the action of a changing number of elementary processes ("coalescence events") with characteristic scale of the order of the temporal fine structure in microwave bursts $(\sim 0.5-3 \mathrm{~s})$. Even scales in the millisecond range, whose occurrence is characteristic of the decimetric range but questionable for the millimeter-centimeter wavelength range, can be explained if the coalescence is restricted to small volumes. The succession of 
the coalescence events determines the envelope of the energetic particle production and microwave burst flux profile. It is known that the coalescence is also a promising candidate for direct particle acceleration in flares.

The circuit model is particularly suited to understanding a hierarchy of timescales. In the frame of this model short timescales $(\sim 1-10 \mathrm{~s})$ correspond to the characteristic time of the flute instability for prominence matter penetrating into the current channel, $\tau_{\mathrm{fl}}$. Subflare timescales $(\sim 10-100 \mathrm{~s})$ are related to the heating of this matter in the current channel. The longest timescale, given by the scale of current dissipation, $\tau_{0} \sim 500-5000 \mathrm{~s}$, corresponds to the duration of the flare process.
These findings show that there is a need for still more detailed studies of hierarchic burst time structures based on extended nonlinear time series analyses (like wavelet analyses) opening new insights into the temporal development of flare processes.

The authors acknowledge fruitful discussions with $U$. Schwarz, A. Tlamicha, and S. Urpo. Useful remarks by an anonymous referee are gratefully acknowledged. This work was supported by grants of the Deutsche Agentur für Raumfahrtangelegenheiten (DARA), nos. 50 QL 92085 and 50 QL 92098 .

\section{REFERENCES}

Alfvén, H., \& Carlquist, P. 1967, Sol. Phys., 1, 220

Aschwanden, M. J., Benz, A. O., \& Schwartz, R. A. 1993, ApJ, 417, 790

Batchelor, D. A. 1987, in Rapid Fluctuations in Solar Flares, ed. B. R.

Dennis, L. E. Orwing, \& A. L. Kiplinger (NASA CP-2449), 35

- 1989, ApJ, 340,607 1990, ApJS, 73, 131

Batchelor, D. A., Crannell, C. J., Wiehl, H. J., \& Magun, A. 1985, ApJ, 295,258

Benz, A. O. 1985, Sol. Phys., 96, 357

Bhattacharjee, A., Brunel, F., \& Taiima, T. 1983, Phys. Fluids, 26, 3332

Brown, J. C., Melrose, D. B., \& Spicer, D. S. 1979, ApJ, 228, 592

Covington, A. E. 1958, JRASC, 52, 161

Cowling, T. G. 1957, Magnetohydrodynamics (New York: Interscience), 107

Dahlburg, R. B., Antiochos, S. K., \& Zang, T. A. 1991, ApJ, 383, 420

de Jager, C., \& de Jonge, G. 1978, Sol. Phys., 58, 127

Holman, G. D. 1985 , ApJ, 293, 584

Holman, G. D., \& Benka, S. G. 1992, ApJ, 400, L79

Hurford, G. J., Marsh, K. A., Zirin, H., Kaufmann, P., \& Strauss, F. M. 1979, BAAS, 11, 678

Kaufmann, P., Correia, E., Costa, J. E. R., \& Zodi Vaz, A. M. 1986, A\&A, 157,11

Kaufmann, P., Strauss, F. M., Opher, R., \& Laporte, C. 1980, A\&A, 87, 58

Kliem, B. 1988, in Reconnection in Space Plasma, vol. 2, ed. T. D. Guyenne \& J. J. Hunt (ESA SP-285) (Noordwijk: ESA), 117 1994, ApJS, in press

Krüger, A. 1979, Introduction to Solar Radio Astronomy and Radio Physics (Dordrecht: Reidel)
Krüger, A., Aurass, H., Kliem, B., \& Urpo, S. 1987, Proc. 10th European Regional Astron. Meeting of IAU, Publ. Astron. Inst. Czechoslovakian Acad. Sci., 66, 245

Krüger, A., Kliem, B., \& Hildebrandt, J. 1988, in Reconnection in Space Plasma, vol. 2, ed. T. D. Guyenne \& J. J. Hunt (ESA SP-285) (Noordwijk: ESA), 169

Kundu, M. R., \& Vlahos, L. 1982, Space Sci. Rev., 32, 379

Leboueuf, J. N., Tajima, T., \& Dawson, J. M. 1982, Phys. Fluids, 25, 784

Pritchett, P. L., \& Wu, C. C. 1979, Phys. Fluids, 22, 2140

Pustilnik, L. A. 1973, AZh, 50, 1211

Roberts, B., Edwin, P. M., \& Benz, A. O. 1984, ApJ, 279, 857

Sakai, S. I., \& Ohsawa, Y. 1987, Space Sci. Rev., 46, 113

Sturrock, P. A., Kaufmann, P., Moore, R. L., \& Smith, D. F. 1984, Sol. Phys., 94, 341

Taijima, T., Brunel, F., \& Sakai, J. 1982, ApJ, 258, L45

Tajima, T., Sakai, J., Nakajma, T., Kosugi, T., Brunel, F., \& Kundu, M. R. 1987, ApJ, 321, 1031

Takakura, T., Kaufmann, P., Costa, J. E. R., Degaonkar, S. S., Ohki, K., \& Nitta, N. 1983, Nature, 302, 317

Tlamicha, A., Ambrožová, Z., \& Sigmund, T. 1991, Publ. Astron. Inst. Czechoslovakian Acad. Sci. No. 78

Urpo, S., Pohjolainen, S., \& Teräsranta, H. 1992, Helsinki University of Technology, Metsähovi Research Station Report Series A, 12

Zaitsev, V. V., \& Stepanov, A. V. 1989, Soviet Astron. Lett., 15, 154 1991, AZh, 68, 384 1992, Sol. Phys., 139, 343 